\title{
Etude des facteurs associés à la non compliance au traitement de masse ciblant les Schistosomiases dans le District Sanitaire de Bougouni, Bankass et Tominian
}

\section{Study of factors associated with non-compliance with mass treatment targeting Schistosomiasis in the Health District of Bougouni, Bankass and Tominian}

\author{
Traoré $\mathrm{NK}^{1}$, Coulibaly $\mathrm{Yl}^{2}$, Dolo $\mathrm{H}^{3}$, Coulibaly $\mathrm{CA}^{4}$, Sangho $\mathrm{H}^{5}$, Doumbia $\mathrm{S}^{6}$
}

(1) (4) (5) Université des Sciences, des Techniques et des Technologies de Bamako, FMOS/DERSP, Mali

(2) (3) MRTC : Malaria Research and Training Center

(6) FMOS : Faculté de Médecine et d'Odontostomatologie

\section{Résumé}

Introduction : La schistosomiase constitue un enjeu de santé publique d'envergure mondiale, affectant le plus souvent les enfants, elle semble être liée au développement et l'augmentation des réseaux d'irrigation. L'efficacité des traitements de masse (TDM) ciblant les schistosomiases reste compromise par la non compliance au Mali. L'objectif était d'étudier les facteurs associés à la non compliance au TDM dans trois districts sanitaire : Bougouni, Bankass, et Tominian. Matériel et méthodes : II s'agissait d'une étude transversale avec analyse secondaire des données de 2277 participants âgés de 5 14 ans et/ou leurs parents issues de l'enquête réalisée de juin à décembre 2017 dans les districts sanitaires de Bankass, Bougouni et Tominian. La non compliance a été évaluée en utilisant une analyse uni et multi variée (régression logistique) avec le logiciel STATA version13. Résultats : La religion ( $p=0,0001)$, la profession du père $(p=0,006)$, la peur des effets secondaires $(p=0,001)$ et la présence d'hématurie terminale $(p=0,001)$ ont été trouvées statistiquement associées à la non compliance. En terme de contribution au model, la religion explique plus la non compliance, suivie de la peur des effets secondaires avec respectivement $43,79 \%, 18,79 \%$, le sexe contribue moins avec seulement 1,09\%. Conclusion : La religion, la profession du père, la peur des effets secondaires et la présence d'hématurie terminale étaient des facteurs statistiquement associés à la non compliance. Une intervention basée sur l'éducation sanitaire serait nécessaire afin de minimiser le taux de non compliance au TDM ciblant les schistosomiases.

Mots clés : Non compliance - Traitement de masse Schistosomiase- Bougouni - Bankass-Tominian.

\section{Summary \\ Introduction: Schistosomiasis is a global public health issue, often affecting children, of development and expansion of irrigation systems. The effectiveness of mass treatments (CT) targeting schistosomiasis remains compromised by non-compliance in Mali. The objective of studying the factors associated with non-compliance with CT in the health districts of Bankass, Bougouni and Tominian. Material and methods: Analysis of cross- sectional with secondary analysis of data from 2.277 participants aged 5 to 14 years and / or their parents were registered so that they were registered from December to}

December 2017 in the districts sanitary facilities of Bankass, Bougouni and Tominian. The noncompliance was tested using logistic regression analysis with the STATA version13 software. Results: Result: Religion ( $p=$ 0.0001 ), father's occupation ( $p=0.006$ ), fear of side effects $(p=0.001)$ and presence of terminal hematuria ( $p$ $=0.001$ ) were found to be statistically associated with noncompliance. In terms of contribution to the model, the religion explains more the noncompliance, followed by the fear of the side effects with respectively $43.79 \%, 18.79 \%$, the sex contributes less with only $1.09 \%$. Conclusion: Religion, the father's profession, fear of side effects and the presence of terminal hematuria were factors statistically associated with noncompliance. An intervention based on health education would be needed to minimize the rate of non-compliance to MDA targeting schistosomiasis.

Key words: noncompliance; mass drug administration; schistosomiasis; Bougouni; Bankass; Tominian.

Introduction : La schistosomiase est une maladie parasitaire provoquée par des vers plats appelés schistosomes, elle constitue un enjeu de santé publique d'envergure mondiale, car c'est la seconde endémie mondiale après le paludisme (1). Elle est classée parmi les Maladies Tropicales Négligées (MTN) car très répandue et confinée dans les régions en développement ; elle est directement liées à l'extension des réseaux d'irrigation (eaux), et sévissant en foyers sur un mode endémo-épidémique (2).

Au Mali, la schistosomiase est présente dans presque toutes les régions avec des prévalences variables selon les zones éco-climatiques. Les régions les plus touchées sont : Kayes, Koulikoro, Ségou, Mopti, Bamako et Sikasso (Sélingué) (3).

Dans ces localités, plus de 10 millions de personnes sont exposées à la maladie. La stratégie retenue par l'Organisation Mondiale de la Santé (OMS) pour lutter contre les schistosomiases est : le traitement de masse (chimio prévention), l'assainissement du milieu, la lutte contre les mollusques, le changement de comportement, l'approvisionnement en eau potable et l'éducation sanitaire (4). L'OMS a recommandé à tous les Etats membres dont le mali à faire une administration régulière d'une chimiothérapie à au moins $75 \%$ des enfants d'âge scolaire exposés (couverture thérapeutique) ; de traiter les adultes à risque élevé du fait de leurs activités, de traiter $100 \%$ des villages endémiques (couverture géographique); d'atteindre au moins $5 \%$ de l'intensité de l'infection (forts excréteurs) dans les sites sentinelles. 
Au Mali les stratégies de contrôle adoptées sont en phase avec celle de l'OMS à savoir la chimio prévention, la formation du personnel socio sanitaire, l'éducation sanitaire et l'assainissement du milieu. En effet, le traitement de masse au Praziquantel bien conduit, permet de réduire la prévalence et l'intensité de l'infection. Ainsi, à long terme, elle contribue à réduire le nombre d'œufs dans le milieu environnant afin de réduire la morbidité et la mortalité liées à la schistosomiase.

Les couvertures thérapeutique et géographique sont à plus de 95\% en 2017 selon le rapport annuel du Programme National de Lutte contre les Schistosomiases et les Géo-Helminthiases.

Cependant, malgré cette bonne couverture, le traitement de masse contre les schistosomiases connait certaines difficultés dont la plus fréquente est la réticence au traitement (non compliance) dans certaines communautés.

Un non compliant est une personne qui a refusé le traitement malgré la sensibilisation ou le conseil lors d'une campagne de TDM ou qui a délibérément refusé d'avaler le médicament (non-conformité).

Des cas de non compliance sont régulièrement rapportés par presque tous les districts lors des campagnes de distribution de masse intégrée contre les MTN. Dans la région de Sikasso le taux de non compliance rapporté était à 16,3\% en 2014 ; la plupart de ces non compliances serait en rapport avec le traitement de masse contre la schistosomiase (5).

Au regard de toutes ces difficultés liées au traitement de masse ciblant les schistosomiases, nous nous sommes proposés d'étudier les facteurs liés à la non compliance au traitement de masse dans les districts sanitaires de Bougouni, Bankass et Tominian. Elle vise à faire une analyse secondaire des données concernant les facteurs associés à la non-compliance au traitement de masse contre les schistosomiases dans les trois districts sanitaires concernés.

Cette étude pourra fournir des évidences scientifiques qui aideront les acteurs des Programmes Nationaux de lutte contre les Schistosomiases à améliorer leurs couvertures thérapeutiques et aussi permettre une plus grande adhésion des communautés aux activités de lutte contre les schistosomiases.

Matériel et Méthodes : L'étude a été conduite dans les districts sanitaires de Bougouni, Bankass et Tominian portant sur 2277 enfants et/ou leurs parents. II s'agissait d'une étude transversale déroulée de janvier à septembre 2019 sur les données des enfants âgés de 5-14 ans et/ou leur parent ayant participé au traitement de masse ciblant les schistosomiases de l'année 2017 et ayant donné leur consentement éclairé ou assentiment. L'étude a été conduite après l'approbation du protocole par le comité d'éthique de la FMOS. Les données provenant de l'étude réalisée de juin à décembre 2017 ont été utilisées pour faire l'analyse secondaire. Un échantillonnage non probabiliste de convenance a été utilisé. L'objectif était de limiter l'échantillon aux 500 premiers enfants volontaires dans le groupe d'âge 5-14 ans et/ou les parents des enfants de 5-14 ans plus jeune. Les enfants âgés de 5-14 ans ont répondu aux questions concernant leurs parents, les parents ont intervenu seulement si les enfants n'étaient pas en mesure de répondre à la question qui lui a été posé. Dans chacune de ces aires de santé, deux villages ont été choisis à savoir le village qui avait le plus fort taux en TDM ciblant les schistosomiases et le village qui avait le plus faible taux en TDM ciblant les schistosomiases. Cette approche nous a fait retenir deux aires de santé et quatre villages dans chacun des trois districts sanitaires de l'étude. Les enquêteurs ont été formés environ une semaine avant la sortie sur le terrain sur la technique d'administration du questionnaire à l'étude primaire réalisée de juin à décembre 2017. Le choix des villages et la technique de collecte des données sont issus également de l'étude primaire réalisée de juin à décembre 2017 dans les districts sanitaires de Bankass, Bougouni et Tominian. La présente étude n'a pas réalisé de collecte des données. La collecte des données a été possible grâce à une enquête de ménage permettant de recueillir des informations sur les cibles concernées. Compte tenu de la période scolaire et de l'âge scolaire de la cible, l'enquête a été majoritairement réalisée dans les écoles. Les questionnaires ont été élaborés et validés en travaux de groupe avec l'équipe cadre des districts et pré-testés dans l'un des villages de l'aire de santé de Tioribougou du district sanitaire de Kolokani. Les données recueillies ont été saisies en utilisant l'application Microsoft Office Excel 2007 dans l'étude primaire. Une analyse secondaire des données a été réalisée en utilisant le logiciel STATA (version 13) dans cette étude. Elle a consisté à faire la fréquence des variables explicatives en fonction de la variable dépendante en analyse univariée et multi variée.

Une analyse univariée été effectué pour déterminer les variables significativement liées à la variable dépendante, par la suite ces variables seront introduites dans le model multi varié.

Une analyse multiple a été réalisée en utilisant la régression logistique pour connaitre les variables statistiquement liées à la non compliance et la contribution de chaque variable dans le modèle. Le seuil de significativité utilisé était 0.05 .

Résultats : L'étude a porté sur les données de 2277 enfants âgés de 5-14 ans et/ou parents d'enfant venant des districts sanitaires de Bankass, Bougouni et Tominian et ayant participé au traitement de masse ciblant les schistosomiases de l'année 2017.

Les enfants âgés de 5-14 ans ont été répartis en trois tranches d'âges (5-7 ans, 8-10 ans et 10-14 ans). 
Les garçons étaient les plus représentés avec $52,7 \%$ contre $47,3 \%$ pour les filles, les enfants 8-10 ans étaient les plus représentés soit 896 enfants majoritairement de niveau de scolarisation primaire (Tableau I).

La profession de tous les parents d'enfants âgés de 5-14 ans ayant participé à l'étude a été donnée. Les cultivateurs étaient les plus représentées pour les pères avec $87,04 \%$ et les ménagères pour celle des mères avec $94,51 \%$ (Tableau II).

Concernant la connaissance de la schistosomiase, elle représentait plus de la moitié des enfants âgés de 5 à 14 ans enquêtées soit $53,15 \%$ qui connaissaient la schistosomiase comme une maladie, les enfants qui présentaient l'hématurie pendant la période d'étude représentaient $10 \%$ et par rapport à l'attitude, $15,64 \%$ avaient peur des effets indésirables du praziquantel (Tableau III).

Les rumeurs représentaient la majorité des motifs de non compliance avec $25,04 \%$, suivi du refus des parents avec 20,36\%, Cependant, 0,16\% des enfants âgés de 5-14 ans refusait le traitement de masse à cause de la grossesse (Tableau IV).

II ressort dans la relation entre la non compliance et les caractéristiques socio démographiques des enfants âgés de 5-14 ans, que le sexe qu'il soit masculin ou féminin avec respectivement (OR 0,22 et OR 0,$19 ; p=0,214)$, n'a pas de lien statistiquement significatif avec la non compliance ainsi que l'âge $(P=0,275)$, et le niveau de scolarisation $(p=0.061)$.

Le niveau d'instruction primaire avait plus de risque $(p=0,001, I C=0,51-0,83$ ) que les non scolarisés (Tableau V).

Dans la relation entre non compliance et caractéristiques socio démographiques de parents ; ce sont les croyances religieuses et la profession des pères qui ont été statistiquement associées de façon significative avec des $p<0,05$.

Ainsi les parents ayant des croyances chrétiennes $(p=0,001, I C=0,12-0,45)$ et animistes $(p=0,001, I C=0,14$ 0,42 ) sont plus enclins d'être non compliants que les croyances musulmanes.

Les pères d'enfants cultivateurs étaient plus enclins d'être non compliants que ceux effectuant une autre profession (Tableau VI).

La peur des effets secondaires $(p=0,001)$ et la présence d'hématurie terminale $(p=0001)$ ont été trouvées statistiquement associées à la non compliance tandis que la connaissance de la schistosomiase $(p=0,661)$ et la connaissance du mode de transmission $(p=0,255)$ n'avaient pas de lien statistiquement significatif avec la non compliance $(p>0,05)$.

Les variables n'ayant pas de lien statistiquement significative avec la non compliance étaient entre autres la connaissance de la schistosomiase $(p=0,661)$, la connaissance du mode de transmission $(p=0,255)$ (Tableau VII).
L'hématurie avait un lien avec la non compliance, les enfants qui ne présentaient pas d'hématurie terminale au moment de l'enquête étaient moins susceptibles d'être non compliants en comparaison avec ceux qui avaient de l'hématurie ( $p=0,001)$ (Tableau VIII).

Les enfants ayant complété le niveau d'instruction primaire avaient 0,35 fois moins de risque d'être non compliants aux TDM que ceux n'ayant pas été scolarisés $(p=0,001, I C=0,51-0,83)$.

Les personnes qui n'avaient pas d'hématurie terminale au moment de l'enquête étaient 0,49 fois moins susceptible d'être non compliants en comparaison avec celles qui avaient une hématurie terminale $(p=0,001 ; I C=0,36-0,72)$ (Tableau IX)

L'analyse explicative a montré que les croyances religieuses ont un effet significatif sur la non compliance du TDM. En effet, les chrétiens ( $p=0,000, I C=0,12-0,45)$ et les animistes $(p=0,000, I C=0.14-0,42)$ étaient 0,76 fois moins susceptibles d'être non compliants au TDM que les musulmans. Les enfants qui avaient leur père effectuant d'autre tâche (orpailleur, enseignant, agent de santé, etc...) comme activité professionnelle principale étaient 2,21 fois plus susceptible d'être non compliants au TDM ciblant les schistosomiases que ceux ayant le père cultivateur $(p=0,001 ; I C=1,45-3,38)$.

La régression a également permis de montrer que certaines variables n'avaient pas de lien statistiquement significatif avec la non compliance au TDM ciblant les schistosomiases. II s'agissait de la profession de la mère, la connaissance de la schistosomiase, le sexe et enfin la tranche d'âge des enfants n'avaient pas d'effet statistiquement significatif sur la non compliance au TDM contre les schistosomiases (Tableau X).

En termes de contribution au modèle, la religion explique le plus la non compliance au TDM ciblant les schistosomiases avec $43,35 \%$, ensuite, la peur des effets secondaires, le niveau de scolarisation, la profession du père, la présence d'hématurie terminale, la profession de la mère, la connaissance de la schistosomiase, la tranche d'âge et enfin le sexe avec 1,09\% (Tableau XI).

\section{Discussion}

La présente étude a permis d'observer une prédominance du sexe masculin avec $52.7 \%$, par ailleurs le sexe n'avait pas de lien statistiquement significatif avec la non compliance $(p=0,214)$. Ceci pourrait s'expliquer par le fait que l'enquête a été réalisée majoritairement dans les écoles et le taux de scolarisation des garçons est le plus souvent élevé que celui des filles. L'étude réalisée par Inobaya MT, et al en 2018 aux Philippines (7) montre au contraire que le sexe masculin était plus susceptible d'être non compliant $(O R=1,67, p=0,033)(7)$. Celle réalisée par Muhumuza S et al. en 2013 en Ouganda.(8) montre que le sexe féminin était le plus représenté avec $55,0 \%(8)$ et celui de Dabo $\mathrm{A}$, et al. réalisée à Bamako au Mali en 2013 (9), montre que le sexe féminin était dominant avec $(52,2 \%)$ surtout dans la tranche d'âge 15 à 34 ans $(p<0,01)(9)$. 
L'étude portait sur les enfants âgés de 5-14ans et/ou parents ; la tranche d'âge 8-10 ans était la plus représentée chez les enfants âgés de 5-14 ans avec $39 \%$. Par ailleurs l'âge n'avait pas d'effet statistiquement significatif sur la non compliance $(p=0,275)$.

Comparativement à l'étude Sud-Africaine qui trouve que la tranche d'âge des 3-9ans est la plus représentée(10) Les croyances religieuses $(p=0,0001)$, la profession $d u$ père $(p=0,006)$; la peur des effets secondaires $(O R=$ 0.19 , test de chi2 de Pearson $=32.0512 ; p=0,001)$ et la présence d'hématurie terminale $(p=0001)$ avaient un lien statistiquement significatif avec la non compliance avec des $p$ inférieurs à 0,05 . Ainsi les parents ayant des croyances chrétiennes et les animistes étaient plus enclins d'être non compliants aux TDM que les musulmans. Cette différence est statistiquement significatif avec les $p<0.05(p=0,001, I C=0,12-0,45$ pour les chrétiens et $p=0,001, I C=0.14-.42$ ) pour les animistes. Le niveau d'instruction primaire était plus succeptible d'être non compliant aux TDM que les non scolarisés, cette différence était statistiquement significatif ( $p=0,001$, $\mathrm{IC}=0,51-0,83$ ). Ceci pourrait s'expliquer par le fait que les enfants au niveau primaire n'ayant pas atteint une certaine maturité et une conscience assez bonne leur permettant de connaitre les avantages du TDM sur leur santé afin de prendre une décision d'accepter ou de refuser un médicament. Le plus souvent à cet âge les enfants acceptent de prendre les médicaments à cause du goût relativement sucré ou sous la pression des parents ou des enseignants. Cependant l'âge ( $P=0,275)$, le niveau de scolarisation $(p=0.061)$; la connaissance de la schistosomiase $(p=0,661)$, la connaissance du mode de transmission $(p=0,255)$ n'avaient pas de lien statistiquement significative avec la non compliance.

Autre profession des pères avaient 2,21 fois plus de risques d'être non compliante aux TDM que les cultivateurs. La différence était statistiquement significative $\quad(p=0,001, \quad I C=1,45-3,38)$.Ceci pourrait s'expliquer par le fait que les cultivateurs semblent être les plus exposés au risque de contracter la schistosomiase compte tenu de leurs activités que les parents effectuant autre profession. Contrairement à l'étude réalisée par Inobaya MT, et al. aux Philippines en 2018 (7) qui montre que les agriculteurs sont statistiquement associés à la non-compliance $(O R=0,62, p=0,038)$. L'étude d'Inobaya MT, et al aux Philippines en 2018 aborde dans le sens que la peur des indésirables du Praziquantel est associée à une cote plus élevée à la non-compliance (OR $=1,94, p=0,021)(7)$. Les rumeurs, le refus des parents et la peur des effets indésirables étaient les plus cités comme motifs de la non compliance avec respectivement $25,04 \%, 20.36 \%$ et $14.54 \%$. L'étude réalisée par Inobaya MT, et al en 2018 aux Philippines (7) montre aussi que les rumeurs $(O R=1,45, p=0,037)$ et que les fausses idées sur le praziquantel $(O R=2,15, p=0,021)$, étaient statistiquement associées à une cote plus élevée à la nonconformité.
La présente étude a retrouvé la peur des effets secondaires comme étant le $3^{\mathrm{e}}$ motif de non compliance (23\%), contrairement à l'étude ougandaise de 2013 ou elle venait en première place avec $72 \%$ (11), l'étude de Muhumuza S et al. en 2013 en Ouganda(12) montre que la peur des effets secondaires du Praziquantel était citée comme la raison majeure de non compliance au cours de laquelle $72 \%$ des participants n'ont pas pris le médicament (12).

Le taux global de non compliant au TDM ciblant les schistosomiases était $16.91 \%$. Ce taux se trouve dans la marge acceptable de l'OMS qui recommande à tous les Etats membres d'atteindre une couverture thérapeutique d'au moins $75 \%$ et une couverture géographique de $100 \%$. Cependant ce taux était jugé non satisfaisant étant donné que la couverture géographique était à $72,86 \%$ en 2017. L'étude réalisée par Inobaya MT, et al aux Philippines en 2018 (7) montre que ce taux était à 27\% et celle de Muhumuza $S$ et al. en 2013 en Ouganda(12) trouve 28,2\%(11). L'étude de Dabo $\mathrm{A}$, et al. (9) réalisée à Bamako au Mali en 2013 trouve seulement 1,7\% de non compliant.

Les enfants qui n'avaient pas d'hématurie terminale au moment de l'enquête avaient moins de risque d'être non compliant comparativement à ceux qui avaient une hématurie terminale, cette différence était statistiquement significative $(p=0,001)$

La connaissance de la schistosomiase par les enfants et/ou les parents des enfants âgés de 5-14 ans n'avait pas d'effet statistiquement significatif sur la non compliance au TDM ciblant les schistosomiases (OR $0,21, p=0,255, I C=0,99-1,62$ ).

Cependant, les enfants qui ne connaissaient pas la schistosomiase comme maladie avaient 1,27 fois plus de risque d'être non compliant que ceux qui la connaissent comme une maladie mais cette différence n'était pas significatif $p=0,061$.

Ce résultat est contraire à celui de l'étude réalisée à Philippine en 2018 par Inobaya MT, et al. (7) ou les répondants qui pensaient que la schistosomiase avait été contractée à la suite d'une défécation à l'air libre et d'un mauvais assainissement $(O R=1,41, p=0,015)$ et en buvant de l'eau sale $(O R=2,09, p=0,001)$ étaient plus susceptibles de refuser un traitement(7).

Une étude réalisée dans les îles L. Victoria en Ouganda par Kabatereine et al(12) en 2014 montre que, bien que le niveau d'éducation semble être associé aux connaissances, l'association n'était significative que parmi les diplômés de l'enseignement supérieur par rapport aux analphabètes $(p=0,02)(4)$.

Cette étude s'est trouvée cependant limitée au regard de certaines difficultés rencontrées comme le manque d'information sur les variables relatives aux parents telles que l'âge, le niveau de scolarisation et le revenu mensuel. Elle a été également limitée dans la réalisation de l'analyse des sous-groupes (l'analyse par district, par village et par aires de santé), ainsi que l'analyse de la corrélation entre les différents variables. 
Conclusion : La religion, la profession du père, la peur des effets secondaires et la présence d'hématurie terminale étaient des variables statistiquement associés à la non compliance. Une intervention basée sur l'éducation sanitaire pour lutter contre les rumeurs et le refus des parents serait nécessaire afin de minimiser le taux de non compliance au TDM ciblant les schistosomiases.

\section{Références}

1. WHO. World Health Organization. WHO Media centre. OMS | Schistosomiase (bilharziose) [Internet]. $2017 . \quad$ Disponible sur: http://www.who.int/mediacentre/factsheets/fs115/fr/

2. M. Sacko, G. Coulibaly, A.D. Keita, A. Landouré, M. Diallo, A.H. Traoré \& M. Traoré : Epidémiologie et morbidité des Schistosomes dans la haute vallée du fleuve Sénégal au Mali.

3. PNLSH. Rapport d'activité annuel 2014/Programme National de Lutte contre les Schistosomiases et les Géohelminthiases. Bamako/Mali: Programme National de Lutte contre les Schistosomiases et les Géo-Helminthiases; 2014 déc. Report No.: 01.

4. Organisation mondiale de la Santé, 1211 Genève 27, Suisse ; Schistosomiase (Bilharrziose) OMS.

6. I Dicko, YI Coulibaly, SY Coulibaly, H Dolo, MB Sangare, S Konate, ME Coulibaly, L Soumaoro, AA Diallo, M Sangare, B Dembele, M Dembele, P Hooper, E Ottesen, D Kyelem, TB Nutman, SF Traore AK. Systemic non-compliance: A potential factor inthe Re-emergence of lymphatic filariasis transmission in Sikasso, Mali. Am J Trop Med Hyg. 2014;91(5 SUPPL. 1):518-9.

7. Inobaya MT, et al. Parasit Vectors. 2018. Mass drug administration and the sustainable control of schistosomiasis: an evaluation of treatment compliance in the rural Philippines. Medlines. 2018;

8. Muhumuza S, Olsen A, Katahoire A, Nuwaha F. Uptake of Preventive Treatment for Intestinal Schistosomiasis among School Children in Jinja District, Uganda: A Cross Sectional Study. PLOS [Internet]. 2013 [cité 18 juill 2019]. Disponible sur: [Internet]. 2013;8(5):16. Available from: www.plosone.org

9. Dabo A, Bary B, Kouriba B, Sankaré O, Doumbo 0 . Factors associated with coverage of praziquantel for schistosomiasis control in the community-direct intervention (CDI) approach in Mali (West Africa) [Internet]. 2013 [cité 18 juill 2019]. Disponible sur: http://www.idpjournal.com/content/2/1/11

10. Kundai Magaisa I; Myra Taylor II; Eyrun F. Kjetland II , III ; Panjasaram J. Naidoo. Examen de la lutte contre la schistosomiase en Afrique du Sud. S Afr j sci vol111 n11-12 Pretoria Nov/Dec 2015 [Internet]. déc 2015 [cité 18 juill 2019]; Disponible sur: http://dx.doi.org/10.17159/sajs.2015/20140427

11. Muhumuza S, Olsen A, Katahoire A, Nuwaha F. Uptake of Preventive Treatment for Intestinal Schistosomiasis among School Children in Jinja District, Uganda: A Cross Sectional Study. PLOS. 2013;8(5):1-6.
12. Kabatereine et al. Perceptions, attitudes, pratiques et traitements de la communauté vis-à-vis de la schistosomiase dans les îles L. Victoria en Ouganda. BioMed Central 2014 [Internet]. 11 déc 2014 [cité 18 juill 2019]; Disponible sur: https://doi.org/10.1186/1756-05007-900

\section{Liste des tableaux}

Tableau I : Caractéristiques sociodémographiques des enfants âgés de 5-14 ans

\begin{tabular}{lll}
\hline Caractéristiques des enfants & $\mathbf{n}$ & $\%$ \\
\hline Sexe & & \\
$\quad$ Masculin & 1200 & 52,7 \\
$\quad$ Féminin & 1077 & 47,3 \\
Tranche d'âge & & \\
5-7 ans & 690 & 30,3 \\
8-10 ans & 896 & 39,35 \\
11-14 ans & 691 & 30,35 \\
Niveau de scolarisation & & \\
$\quad$ Non scolarisé & 911 & 40,01 \\
$\quad$ Primaire & 1300 & 57,09 \\
$\quad$ Secondaire & 8 & 0,35 \\
Pré scolaire & 5 & 0,22 \\
Ecole coranique & 53 & 2,33 \\
\hline
\end{tabular}

Tableau II : Caractéristiques sociodémographiques des parents d'enfants âgés de 5-14 ans

\begin{tabular}{|c|c|c|}
\hline $\begin{array}{l}\text { Caractéristiques des } \\
\text { parents }\end{array}$ & $\mathbf{n}$ & $\%$ \\
\hline \multicolumn{3}{|l|}{ Profession du père } \\
\hline Cultivateur & 1982 & 87,04 \\
\hline Commerçant & 69 & 3,03 \\
\hline Artisan & 52 & 2,28 \\
\hline Eleveur & 19 & 0,83 \\
\hline Autre $^{*}$ & 155 & 6,81 \\
\hline \multicolumn{3}{|l|}{ Profession de la mère } \\
\hline Ménagère & 2152 & 94,51 \\
\hline Commerçante & 64 & 2,81 \\
\hline Artisan & 19 & 0,83 \\
\hline Autre** & 40 & 1,76 \\
\hline Ne sait pas & 2 & 0,09 \\
\hline
\end{tabular}

Tableau III : Connaissances et attitudes par rapport à la schistosomiase et de la fréquence de l'hématurie dans les districts d'étude

\begin{tabular}{lll}
\hline Variations & $\mathrm{n}$ & $\%$ \\
\hline
\end{tabular}




\section{Compliance \\ Non compliant \\ Compliant}

Connaissance de la

Schistosomiase

$\begin{array}{lll}\text { Oui } & 1207 & 53,15 \\ \text { Non } & 1064 & 46,85 \\ \text { Présence d'hématurie terminale } & & \end{array}$

Présence d'hématurie terminale

$\begin{array}{lcc}\text { Oui } & 243 & 10,72 \\ \text { Non } & 2023 & 89,28 \\ \begin{array}{l}\text { Connaissance du mode de } \\ \text { transmission }\end{array} & & \\ \text { Oui } & 219 & 9,66 \\ \text { Non } & 2048 & 90,34\end{array}$

Peur des effets secondaires Oui

$385 \quad 16,91$

189283,09

$356 \quad 15,64$

\begin{tabular}{lll}
\hline Non & 1920 & 84,36 \\
\hline
\end{tabular}

Tableau IV : Répartition des non compliants selon les motifs de la non compliance dans les trois districts d'étude

\begin{tabular}{lrr}
\hline Motifs de non compliance & $\mathbf{n}$ & $\%$ \\
\hline Rumeurs & 155 & 25,04 \\
Refus des parents & 126 & 20,36 \\
Peur des effets secondaires & 90 & 15,54 \\
Non informé & 67 & 10,82 \\
Supervision de la campagne de & 8 & 1,29 \\
DMM par le personnel Sanitaire & 5 & 0,81 \\
Autres & 3 & 0,48 \\
Nombre élevé de comprimé à avaler & 1 & 0,16 \\
Grossesse & & \\
\hline
\end{tabular}

Tableau V : Relation entre la non compliance et certaines caractéristiques sociodémographiques des enfants âgés de 5-14ans dans les trois districts d'étude

\begin{tabular}{|c|c|c|c|}
\hline & $\begin{array}{c}\text { Compliant } \\
n(\%)\end{array}$ & $\begin{array}{c}\text { Non compliant } \\
n(\%)\end{array}$ & $p$ \\
\hline \multicolumn{4}{|l|}{ Sexe des enfants } \\
\hline Masculin & $986(82,17)$ & $214(17,83)$ & 0,214 \\
\hline Féminin & $906(84,12)$ & $171(15,88)$ & \\
\hline \multicolumn{4}{|c|}{ Tranche d'âge des enfants } \\
\hline 5-7 ans & $577(83,62)$ & $113(16,38)$ & 0,275 \\
\hline $8-10$ ans & $731(81,58)$ & $165(18,42)$ & \\
\hline $11-14$ ans & $584(84,52)$ & $107(15,48)$ & \\
\hline \multicolumn{4}{|c|}{ Niveau de scolarisation des enfants } \\
\hline Non scolarisé & $735(80,68)$ & $176(19,32)$ & $0,109^{*}$ \\
\hline Primaire & $1102(84,77)$ & $198(15,23)$ & \\
\hline Secondaire & $7(87,50)$ & $1(12,50)$ & \\
\hline Pré scolaire & $5(100)$ & $0(0)$ & \\
\hline Ecole coranique & $43(81,13)$ & $10(18,87)$ & \\
\hline
\end{tabular}

* : test exact de Fisher

Tableau VI : Relation entre la non compliance et certaines caractéristiques sociodémographiques des parents enfants âgés de 5-14 ans dans les trois districts d'étude

\begin{tabular}{lccc}
\hline & $\begin{array}{c}\text { Compliant } \\
\mathrm{n}(\%)\end{array}$ & $\begin{array}{c}\text { Non compliant } \\
\mathrm{n}(\%)\end{array}$ & $\mathbf{p}^{*}$ \\
\hline Profession des pères & $\mathbf{1 8 9 2 ( 8 3 , 0 9 )}$ & $\mathbf{3 8 5}(\mathbf{1 6 , 9 1 )}$ & \\
Cultivateur & $1668(84,16)$ & $314(15,84)$ & \\
Commerçant & $54(78,26)$ & $15(21,74)$ & \\
Artisan & $42(80,77)$ & $10(19,23)$ & 0,006 \\
Eleveur & $15(78,95)$ & $4(21,05)$ & \\
Autre* $^{*}$ & $113(72,9)$ & $42(27,1)$ & \\
\hline
\end{tabular}




\begin{tabular}{lccc}
\hline Religion des parents & $1892(83,09)$ & $385(16,91)$ & \\
Musulman & $1443(80,21)$ & $356(19,79)$ & \\
Chrétien & $199(94,76)$ & $11(5,24)$ & 0,0001 \\
Animiste & $244(94,21)$ & $15(5,79)$ & \\
Aucune & $6(66,67)$ & $3(33,33)$ & \\
\hline
\end{tabular}

$p^{*}=$ Test exact de Fisher

Tableau VII : Relation entre la non compliance et la connaissance de la schistosomiase chez les enfants de 5 -14 ans dans les trois districts d'étude.

\begin{tabular}{lccc}
\hline & $\begin{array}{c}\text { Compliant } \\
\mathbf{n}(\%)\end{array}$ & $\begin{array}{c}\text { Non compliant } \\
\mathbf{n}(\%)\end{array}$ & $\mathbf{p}$ \\
\hline Connaissance de la schistosomiase & & & \\
$\quad$ Oui & $999(82,77)$ & $208(17,23)$ & 0,661 \\
$\quad$ Non & $888(83,46)$ & $176(16,54)$ & \\
Connaissance du mode de transmission & & & \\
$\quad$ Oui & $188(85,84)$ & $31(14,16)$ & 0,49 \\
$\quad$ Non & $1696(82,81)$ & $352(17,19)$ & \\
$\quad$ Peur effets secondaire & & & \\
$\quad$ Oui & $259(72,75)$ & $97(27,25)$ & 0,001 \\
$\quad$ Non & $1632(85)$ & $288(15)$ & \\
\hline
\end{tabular}

Tableau IX : Facteurs associés à la non compliance au TDM ciblant les schistosomiases chez les enfants âgés de 514 ans dans les trous districts (Bougouni, Bankass et Tominian)

\begin{tabular}{|c|c|c|c|}
\hline & $\begin{array}{l}\text { Odds Ratio } \\
\text { (OR) }\end{array}$ & $\begin{array}{c}\mathrm{P}>|\mathrm{z}| \\
\text { Likelihood ratio }\end{array}$ & IC/95\% OR \\
\hline \multicolumn{4}{|l|}{ Sexe } \\
\hline Masculin (ref) & 1 & & \\
\hline Féminin & 0,86 & 0,216 & 0,69-1,09 \\
\hline \multicolumn{4}{|l|}{ Groupe d'âge } \\
\hline 5-7 ans (ref) & 1 & & \\
\hline $8-10$ ans & 1,18 & 0,238 & $0,9-1,56$ \\
\hline $11-14$ ans & 0,99 & 0,958 & $0,73-1,35$ \\
\hline \multicolumn{4}{|c|}{ Niveau de Scolarisation } \\
\hline Non scolarisé (ref) & 1 & & \\
\hline Primaire & 0,65 & 0,001 & $0,51-0,83$ \\
\hline Pré scolaire & 1 & & \\
\hline Ecole coranique & 0,8 & 0,558 & $0,38-1,68$ \\
\hline \multicolumn{4}{|c|}{ Peur des effets Secondaires } \\
\hline Oui (ref) & 1 & & \\
\hline Non & 0,46 & 0 & $0,35-0,61$ \\
\hline \multicolumn{4}{|c|}{ Connaissance de la Schistosomiase } \\
\hline Oui (ref) & 1 & & \\
\hline Non & 1,27 & 0,061 & $0,99-1,62$ \\
\hline \multicolumn{4}{|c|}{ présence d'hématurie terminale } \\
\hline Oui (ref) & 1 & & \\
\hline Non & 0,51 & 0 & $0,36-0,72$ \\
\hline
\end{tabular}


Tableau VIII : Relation entre la non compliance et la présence d'hématurie chez les enfants de 5 -14 ans dans les trois districts d'étude.

\begin{tabular}{lccc}
\hline & $\begin{array}{c}\text { Compliant } \\
\mathbf{n}(\%)\end{array}$ & $\begin{array}{c}\text { Non compliant } \\
\mathbf{n}(\%)\end{array}$ & $\mathbf{p}$ \\
\hline Présence d'hématurie terminale & & & \\
Oui & $180(74,07)$ & $63(25,93)$ & \\
Non & $1702(84,13)$ & $321(15,87)$ & 0,001 \\
\hline
\end{tabular}

Tableau X : Facteurs associés à la non compliance au TDM ciblant les schistosomiases chez les parents d'enfants âgés de 5-14 ans dans les trois districts sanitaires de l'étude

\begin{tabular}{lccc} 
& Odds Ratio & $\begin{array}{c}\mathbf{P}>|\mathbf{z}| \\
\text { Likelihood ratio }\end{array}$ & IC/95\% du OR \\
\hline $\begin{array}{l}\text { Profession du père } \\
\text { Cultivateur (ref) }\end{array}$ & 1 & & \\
Commerçant & 1,73 & 0,086 & $0,93-3,23$ \\
Artisan & 1,53 & 0,321 & $0,66-3,52$ \\
Eleveur & 1,17 & 0,79 & $0,38-3,62$ \\
Autre & 2,21 & 0 & $1,45-3,38$ \\
Profession de la mère & & & \\
Ménagère (ref) & 1 & & \\
Commerçante & 1,12 & 0,744 & $0,58-2,16$ \\
Artisan & 0,52 & 0,372 & $0,13-2,18$ \\
Autre & 0,37 & 0,08 & $0,12-1,12$ \\
Ne sait pas & 1 & & \\
Religion & & & \\
Musulman (ref) & 1 & 0 & $0,12-0,45$ \\
Chrétien & 0,24 & 0 & $0,14-0,42$ \\
Animiste & 0,24 & 0,051 & \\
Aucune & 4,52 & & \\
\hline
\end{tabular}

Tableau XI : Contribution des variables au modèle de régression

\begin{tabular}{lc}
\hline Contribution des variables & \% Contribution au model \\
\hline Religion & 43,35 \\
Peur des effets secondaires & 18,79 \\
Niveau de scolarisation & 11,87 \\
Profession du père & 9,09 \\
Présence d'hématurie terminale & 7,85 \\
Profession de la mère & 4,64 \\
Connaissance de la schistosomiase & 1,92 \\
Tranche d'âge & 1,4 \\
Sexe & 1,09 \\
\hline
\end{tabular}

